\title{
Dermoscopy Findings of Hidroacanthoma Simplex
}

\author{
Yota Sato Taku Fujimura Erika Tamabuchi Takahiro Haga \\ Setsuya Aiba \\ Department of Dermatology, Tohoku University Graduate School of Medicine, \\ Sendai, Japan
}

\section{Key Words}

Hidroacanthoma simplex · Dermoscopy $\cdot$ Muskmelon appearance

\begin{abstract}
Hidroacanthoma simplex (HAS), also known as intraepidermal eccrine poroma, is a rare eccrine adnexal tumor that tends to be misdiagnosed as other types of benign skin tumor, including clonal seborrheic keratosis. Notably, HAS is sometimes misdiagnosed and treated by cryosurgery as seborrheic keratosis, which could trigger the later development of porocarcinoma. Therefore, accurate diagnosis of HAS is indispensable for dermatologists to avoid the development of malignant tumors by an unsuitable treatment. In this report, we present the characteristic dermoscopy findings of HAS. Indeed, the dermoscopy findings might be related to the melanin-rich necrotic cells in the epidermis, which are quite different from dermoscopy findings of clonal seborrheic keratosis. As a previous report suggested, it is difficult for a dermatologist to differentiate HAS from clonal seborrheic keratosis by the naked eye. Our findings might be supportive for the early diagnosis of HAS.
\end{abstract}

(C) 2014 S. Karger AG, Basel

\section{Introduction}

Hidroacanthoma simplex (HAS), also known as intraepidermal eccrine poroma, is a rare eccrine adnexal tumor that tends to be misdiagnosed as other types of benign skin tumor, including clonal seborrheic keratosis [1-3]. In contrast to seborrheic keratosis, it is possible for eccrine porocarcinoma to develop from HAS [4]. Notably, HAS is sometimes misdiagnosed and treated by cryosurgery as seborrheic keratosis, which could trigger the development of porocarcinoma in the future. Therefore, accurate diagnosis of HAS is indispensable 
for dermatologists to avoid the development of malignant tumors by an unsuitable treatment. In this report, we present the characteristic dermoscopy findings of HAS.

\section{Case Report}

An 81-year-old Japanese woman visited our outpatient clinic with a 2-year history of a developing, asymptomatic nodule on her left thigh. On her initial visit, physical examination revealed a brown, hyperkeratotic plaque, $26 \times 20 \mathrm{~mm}$ in size on the lateral side of the left thigh (fig. 1a). Dermoscopy of the thinner, brownish part of the tumor revealed characteristic features, namely whitish globular structures surrounded by homogenous, pigmented lines (fig. 1b). At the dark brown, thick part of the tumor, these whitish globular structures were covered with gatherings of the thick, oval keratotic materials, referred to as 'muskmelon appearance' (fig. 1c). A biopsy specimen showed well-demarcated nests within the epidermis (fig. 2a). The nests were mainly composed of cuboidal basaloid cells, which surrounded necrotic cells with abundant melanin granules (fig. 2b). From the above findings, we diagnosed this patient as having HAS. We excised the lesion with a 3-mm surgical margin, and there has been no sign of local recurrence for 1 year.

\section{Discussion}

HAS is a benign eccrine tumor, also reported as an intraepidermal eccrine poroma [1, 2]. Most cases of HAS are clinically misdiagnosed as other types of benign and malignant tumors, including seborrheic keratosis [3]. Notably, though in rare cases, eccrine porocarcinoma develops from HAS [4]. Therefore, accurate diagnosis of HAS is indispensable for dermatologists to avoid the development of malignant tumors by an unsuitable treatment.

Dermoscopy is a valuable, noninvasive, widely used technique that has improved the diagnostic accuracy for skin tumors [5-7]. It allows in vivo observation of the skin with visualization of morphological structures in the epidermis and papillary dermis that are not detectable by the naked eye. For skin cancers, dermoscopy has had a significant impact on the early diagnosis for differentiating malignant skin tumors from those that are benign [6]. Concerning eccrine poroma, 2 types of dermoscopy findings of eccrine poroma were described previously [5]. In addition, Kuo et al. [8] reported dermoscopy findings of 2 cases of pigmented eccrine poroma developing in the dermis, which presents multiple blue-gray oval nests and dark blue dots, and arborizing vessels. More recently, Suzaki et al. [4] reported the dermoscopic feature of eccrine porocarcinoma arising from HAS. In the present report, we added the dermoscopy findings of ordinary HAS that showed more characteristic features. Indeed, these dermoscopy findings, namely the whitish globular structures surrounded by homogenous, pigmented lines, might be related to the melanin-rich necrotic cells in the epidermis, which are quite different from dermoscopy findings of clonal seborrheic keratosis [9]. Because seborrheic keratosis presents a variety of dermoscopic findings, it may be difficult to exactly differentiate some types of seborrheic keratosis from HAS only by dermoscopy [10]. But we would like to suggest that the dermatologist should select the surgical approach to treat the skin tumor with these characteristic dermoscopy findings. Though our present finding is limited because it came from only a single case, this finding might be supportive for the early diagnosis of HAS. 
Sato et al.: Dermoscopy Findings of Hidroacanthoma Simplex

\section{References}

1 Elder DE, Elemenitsas R, Johnson BL Jr, Murphy GF: Lever's Histopathology of the Skin, 10th ed. Philadelphia: Lippencott-Raven, 2008.

-2 Moriwaki K, Tsuruta D, Sowa J, Kobayashi H, Ishii M: Hidroacanthoma simplex with inflammatory dermatosis-like lichenified plaque-type appearance. Dermatology 2005;210:358-359.

-3 Anzai S, Arakawa S, Fujiwara S, Yokoyama S: Hidroacanthoma simplex: a case report and analysis of 70 Japanese cases. Dermatology 2005;210:363-365.

4 Suzaki R, Shioda T, Konohana I, Ishizaki S, Sawada M, Tanaka M: Dermoscopic features of eccrine porocarcinoma arising from hidroacanthoma simplex. Dermatol Res Pract 2010;2010:192371.

-5 Nicolino R, Zalaudek I, Ferrara G, Annese P, Giorgio CM, Moscarella E, Sgambato A, Argenziano G: Dermoscopy of eccrine poroma. Dermatology 2007;215:160-163.

6 Fargnoli MC, Kostaki D, Piccioni A, Micantonio T, Peris K: Dermoscopy in the diagnosis and management of non-melanoma skin cancers. Eur J Dermatol 2012;22:456-463.

7 Fujimura T, Hidaka T, Hashimoto A, Aiba S: Dermoscopy findings of pseudolymphomatous folliculitis. Case Rep Dermatol 2012;4:154-157.

8 Kuo HW, Ohara K: Pigmented eccrine poroma: a report of two cases and study with dermatoscopy. Dermatol Surg 2003;29:1076-1079.

\9 Longo C, Zalaudek I, Moscarella E, Lallas A, Piana S, Pellacani G, Argenziano G: Clonal seborrheic keratosis: dermoscopic and confocal microscopy characterization. J Eur Acad Dermatol Venereol 2013, DOI: 10.1111/jdv.12261 (Epub ahead of print).

10 Johr R, Saghari S, Nouri K: Eccrine porocarcinoma arising in a seborrheic keratosis evaluated with dermoscopy and treated with Mohs' technique. Int J Dermatol 2003;42:653-657. 
Sato et al.: Dermoscopy Findings of Hidroacanthoma Simplex
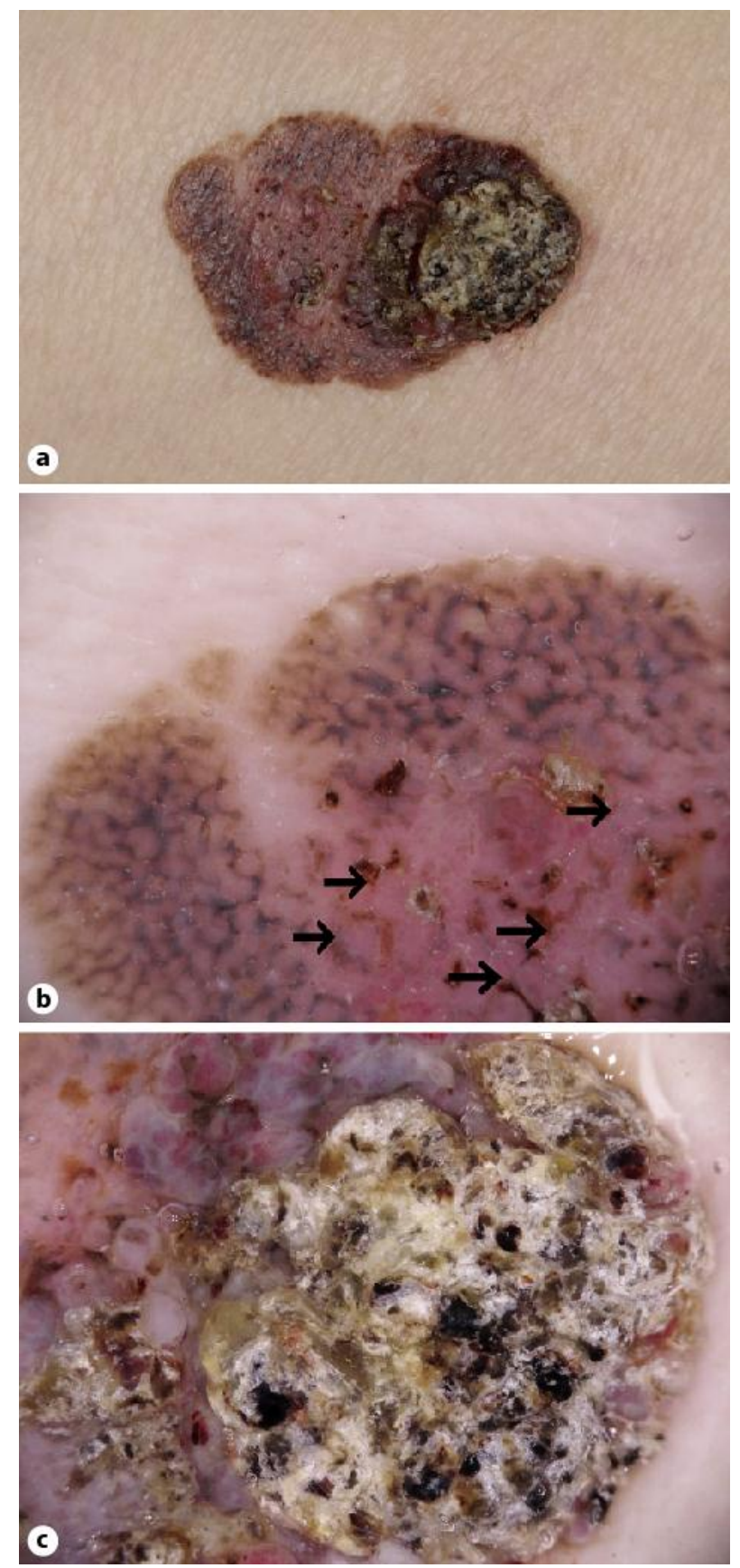

Fig. 1. A brown, hyperkeratotic plaque, $26 \times 20 \mathrm{~mm}$ in size on the lateral side of the left thigh (a). Dermoscopy findings of the thinner, brownish, part of the tumor revealed whitish globular structures (arrows for the representative ones) surrounded by homogenous, pigmented lines (b). At the dark brown, thick part of the tumor, these whitish globular structures were covered with gatherings of the thick, oval keratotic materials, giving them a 'muskmelon appearance' (c). 


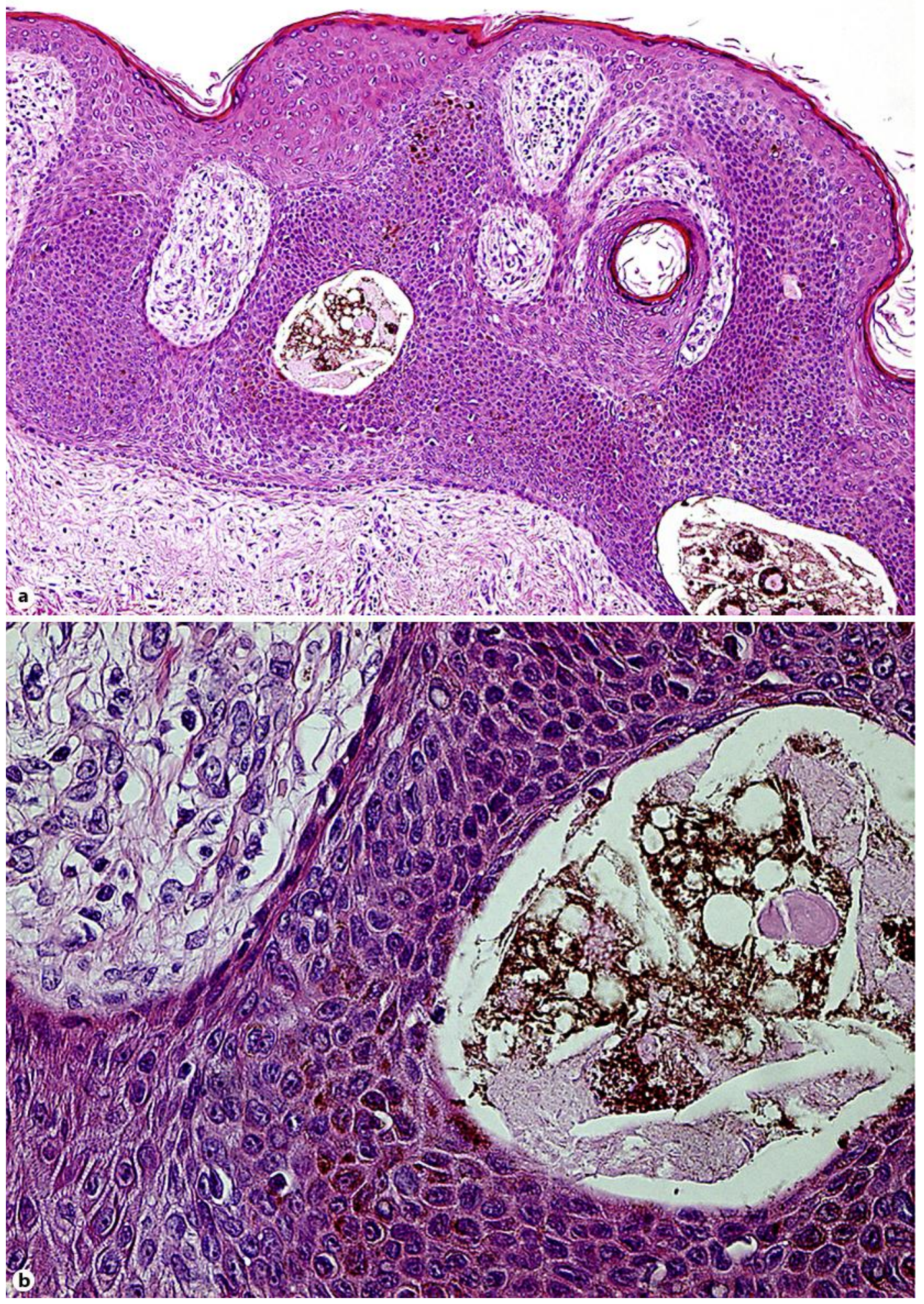

Fig. 2. Well-demarcated nests within the epidermis (a). The nests were mainly composed of cuboidal basaloid cells, which surrounded necrotic cells with abundant melanin granules (b). Original magnification $\times 100(a), \times 400(b)$. 